Dieses Dokument ist eine Zweitveröffentlichung (Verlagsversion) / This is a self-archiving document (Verlagsversion):

Adrian Ehrenhofer, Martin Elstner, Angelos Filippatos, Maik Gude, Thomas Wallmersperger

Window-opener as an example for environment measurement and combined actuation of smart hydrogels

Erstveröffentlichung in / First published in:

SPIE Smart Structures + Nondestructive Evaluation. Online Only, 22.-27.03.2021.

Bellingham: SPIE, Vol. 11587 [Zugriff am: 14.04.2020].

DOI: $\underline{\text { https://doi.org/10.1117/12.2582672 }}$

Diese Version ist verfügbar / This version is available on:

https://nbn-resolving.org/urn:nbn:de:bsz:14-qucosa2-747267 


\section{Window-opener as an example for environment measurement and combined actuation of smart hydrogels}

Ehrenhofer, Adrian, Elstner, Martin, Filippatos, Angelos, Gude, Maik, Wallmersperger, Thomas

Adrian Ehrenhofer, Martin Elstner, Angelos Filippatos, Maik Gude, Thomas Wallmersperger, "Window-opener as an example for environment measurement and combined actuation of smart hydrogels," Proc. SPIE 11587, Electroactive Polymer Actuators and Devices (EAPAD) XXIII, 115870I (22 March 2021); doi: 10.1117/12.2582672

SPIE. Event: SPIE Smart Structures + Nondestructive Evaluation, 2021, Online Only 


\title{
Window-opener as an example for environment measurement and combined actuation of smart hydrogels
}

\author{
Adrian Ehrenhofer ${ }^{\mathrm{a}, \mathrm{b}}$, Martin Elstner ${ }^{\mathrm{c}}$, Angelos Filippatos ${ }^{\mathrm{a}, \mathrm{d}}$, Maik Gude ${ }^{\mathrm{d}, \mathrm{a}}$, and \\ Thomas Wallmersperger ${ }^{\mathrm{b}, \mathrm{a}}$ \\ ånesden Center for Intelligent Materials (DCIM), Technische Universität Dresden, 01069 \\ Dresden, Germany \\ ${ }^{\mathrm{b}}$ Institute of Solid Mechanics, Technische Universität Dresden, 01062 Dresden, Germany \\ ${ }^{\mathrm{c}}$ Covestro Deutschland AG, 51365 Leverkusen, Germany \\ dInstitute of Lightweight Engineering and Polymer Technology, Technische Universität \\ Dresden, 01307 Dresden, Germany
}

\begin{abstract}
An environment is defined by a set of field values, such as temperature, electro-magnetic field, light intensity, air humidity and air composition. Smart materials, such as hydrogels, are able to react to these kinds of stimuli. The spatial and time development of environmental values is governed by transport equations. Hence the reaction, i.e. actuation or sensing, of the smart material can be described based on the same assumptions. The displacement, here swelling and deswelling, of the material depends on the combination of the environmental parameters. Smart materials are called multi-sensitive, when more than one parameter is purposely used (i) to manipulate the material, i.e. as an actuator or (ii) to measure the quantities, i.e. as a (multi-)sensor. However, the material can also perform (iii) the objective of a logic processing unit in addition to (i) and (ii). In the current work, we present a device that realizes this concept: An automatic window opener that senses environmental parameters (light-level and air temperature) and reacts accordingly. The hydrogel material that is included in the simplistic device simultaneously acts as sensor, logic processing unit and actuator.
\end{abstract}

Keywords: Smart/intelligent Materials, Hydrogels, Modeling and Simulation, Multisensitivity, Environments, Logic Behavior

\section{INTRODUCTION: ACTIVE MATERIALS THAT SENSE THE ENVIRONMENT}

Active materials can be designed to sense the environment and to react accordingly. ${ }^{1,2}$ In the current work, we highlight, how a system can be designed to perform an action based on environmental variables. As an example, we provide the task from Figure 1: We imagine a room in a house that heats up during daytime; we want to open the window at night time to vent and cool-down the room. The task is therefore, to detect night-time (low light level) and a high in-door temperature; and to perform an actuation (window opening) if these conditions are met. This task serves as an example and can be extended to include additional environmental variables such as air humidity (inside and outside), air pressure or concentrations of airborne particles. In addition, there may be the need for an override button for special occasions that can be activated manually or as a part of the local Internet-of-Things hub.

For a systematic description of the design process of such a system, we first have to define what is meant by the word environment and how environmental parameters can be understood and modeled. The definition of environment is part of the systematic description of the design requirements in multiple design methods of function-integrative engineering, such as the spiral development approach. ${ }^{3}$ This, as well as the overview of active materials (and especially hydrogels) is given in the introduction sections 1.1 and 1.2). This is followed by a section about material logic, see section 2 . In section 3, the design of the window-opener is presented. The conclusion is drawn in section 4.

Further author information:

Adrian Ehrenhofer: E-mail: Adrian.Ehrenhofer@tu-dresden.de

Angelos Filippatos: E-mail: Angelos.Filippatos@tu-dresden.de 




Figure 1: The task for the window-opener setup is: If it is night and the temperature inside is high, then open the windows. This translates to the logic combination of a low light intensity stimulus $I \downarrow$ and a high temperature stimulus $\vartheta \uparrow$. In the classical approach, two sensors are used and their signals are combined in external electronics. Intelligent materials offer the possibility of direct logical decision inside the material. The material itself acts as an inhibit gate.

\subsection{What is an environment?}

An environment can be understood as a set of physical parameters, such as temperature, air pressure, concentrations of chemical agents or light level. From the continuum mechanical viewpoint, these are tensorial values that are brought into the respective field equation (composed of balance laws, constitutive relations and kinematics) via boundary conditions or sources/sinks. For example, the thermal field for closed systems is denoted by the transient heat transfer equation (composed of the first law of thermodynamics, FouRIER's law and the definition of heat capacity for constant pressure $c_{p}$ )

$$
\rho c_{p} \dot{\vartheta}=\rho r+\lambda \vartheta_{, k k},
$$

where $\vartheta$ is the temperature, $\rho$ the density, $\lambda$ the isotropic heat conduction coefficient and $r$ represents local heat sources/sinks. The time derivative is denoted by () and the spatial derivative with respect to the base coordinates $x_{k}$ is denoted by ()$_{, k}$. Please note that Einstein's summation convention holds true. Besides stimulus transport inside the body, there are two reasons for local changes of a field value inside the body: (i) A stimulus that externally causes a change in the local field variables is introduced by boundary conditions, e.g. a constant temperature or a heat flux. These are Dirichlet and Neumann boundary conditions, or in combined form RoBin boundary conditions. At the same time, the field can be changed by (ii) internal causes, such as exothermal chemical reactions or absorption of light inside the material (BOUGUER-LAMBERT-BEER-law). Both kinds of influences to the field distribution of a physical value can be caused by environmental values. In Figure 2 , a set of tensorial values that can influence a body $V$, is depicted.

There are different definitions for environment in different disciplines. Environmental parameters play a crucial role in most natural sciences and engineering disciplines. The literature ranges from physical influences on museum exhibits, ${ }^{4}$ the spread of airborne infectious agents ${ }^{5}$ to agriculture. ${ }^{6}$ In other disciplines, the term can have another semantic content. For example the term biophysical environment describes biotic and abiotic 


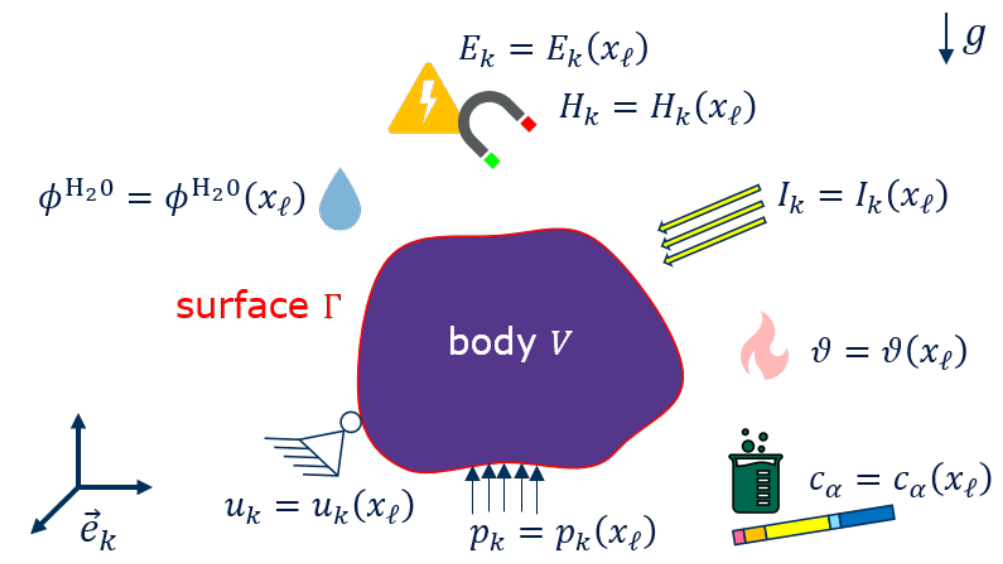

Figure 2: A body $V$, which can be composed by a single (meta-)material or as a composite of multiple materials, is subjected to various environmental parameters. The surface $\Gamma$ is the physical threshold of the body. Influences stem from the mechanical field (displacements $u_{k}$, pressures $p_{k}$ or volume loads due to gravity $\downarrow g$ ); from the chemical field (concentrations $c_{\alpha}$ of a chemical species $\alpha, \mathrm{pH}$ or air humidity $\phi^{\mathrm{H}_{2} \mathrm{O}}$ ), thermal (temperature $\vartheta$ ), electro-magnetic (electric field $E_{k}$, magnetic field $H_{k}$, light intensity $I_{k}$ )

influences on a system and forms the basis of environmental sciences. For the sake of this publication, we focus on the definition of environment of an active material as explained above, i.e. a set of tensorial field variables that affect the body.

\subsection{Active materials}

For a certain active construction (i.e. a body of a single (meta-)material or mixtures in form of composites), the environmental parameters can be subdivided into two groups: (i) sensitivities, i.e. the parameters, that the material/composite is designed to be responsive to and (ii) unintended cross-correlations. Please note that at the same time, the parameters from both (i) and (ii) can have a degradation effect on the material, like corrosion or thermal fatigue.

Structures are called smart or intelligent if they include active elements, which are applied as sensors and/or actuators. $^{7,8}$ This principle is used e.g. in cantilever structures with piezoelectric elements that sense deformation and counteract the movement to reduce vibration. The placement of the logic that controls the actuation is used for differentiating between smart and intelligent behavior: In the case of the cantilever setup, it would be called smart when external computation is performed and intelligent if the electronics are integrated into the cantilever structure itself. ${ }^{7,8}$

Various different classes of (electro-)active materials can be found in literature. ${ }^{9}$ The definition of smart and intelligent is rather diffuse when it is applied to active materials. Smart materials show sensoric and actuatoric behavior is commonly used in literature ${ }^{10}$ and in the previous works by the authors. From the engineer's perspective, both sensing and actuating use the same effect: The shape change is used to measure the stimulus. Another description is that smart materials show a reaction when exposed to a stimulus. ${ }^{11-13}$ However, a block of steel performing thermal expansion is usually not called intelligent. In the context of membranes, smart can also mean with tunable permeation properties. ${ }^{14,15}$

In the current work, we present hydrogels as active materials which provide intrinsic computational capabilities for multiple input stimuli, see figure 3. Smart hydrogels are a promising material class, since they incorporate different stimuli over a broad input range and can be operated at physiological or ambient conditions. ${ }^{16}$ 


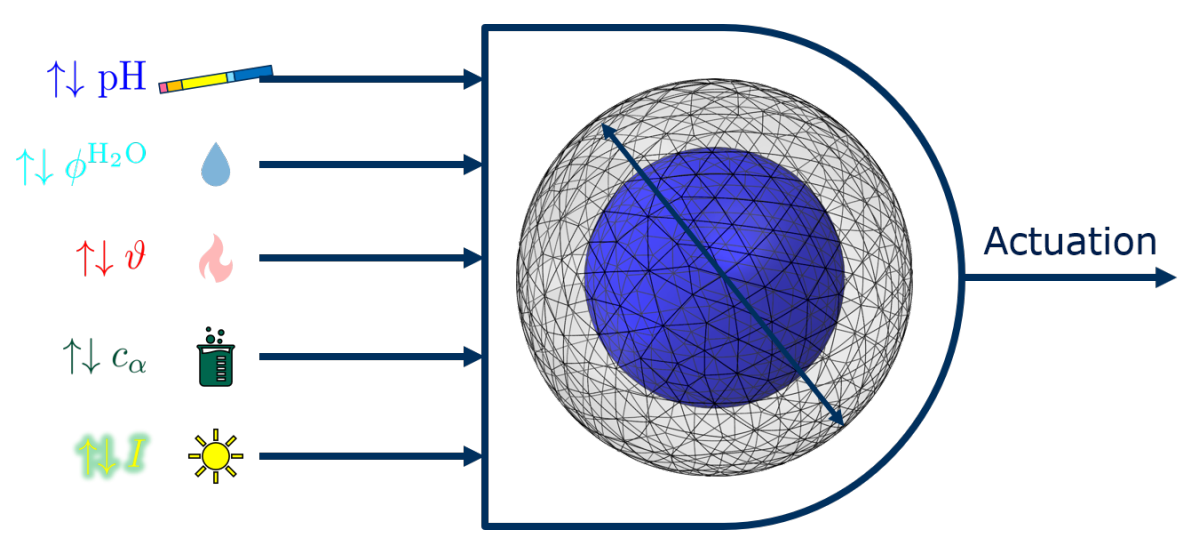

Figure 3: Different input stimuli like the change of $\mathrm{pH}$, temperature, ion concentration or light intensity can lead to a single output. This output is the swelling actuation with the diameter as the chosen parameter.

\section{MATERIAL LOGIC: WHAT MAKES ACTIVE MATERIALS SMART/INTELLIGENT?}

When combinations of environmental parameters - e.g. temperature and light level - are to be measured simultaneously, typically individual sensors for each stimulus are used, see classical approach in Figure 1. Each sensor gives an output signal that is then digitalized through the use of thresholds for high and low value. ${ }^{17,18}$ The Boolean output value 0 or 1 is then fed to signal processing through logic gates for combination. The output of the gate is then amplified and used for an actuator/motor which performs the actuation based on the decision made in the logic gate.

Active materials can be used to simplify this signal transduction process because the logic behavior is integrated in the material itself. ${ }^{19,20}$ We propose that materials that are intelligent provide their own intrinsic logic behavior for multiple input stimuli that can range from simple negation (NOT-gate) to more complex behaviors like implication-gates or inhibition. Also, combinations of logical behavior such as presented in Figure 3 can be achieved. In Figure 1, an application example with light and temperature stimulus input is presented: Only if the light is low and the temperature is high, the actuation is performed. When these conditions are no longer met, the material returns to its initial state.

\subsection{From hydrogels to logic hydrogel elements}

The active response of a hydrogel to multiple stimuli can be characterized as logic gate behavior. The switching of the stimulus, which is the input signal, leads to a volume change (output). In the current section, the fundamentals for the digital description of active multiresponsive materials are presented.

The input signal for a hydrogel can be any stimulus to which the hydrogel is responsive. In our previous work, we focused on the normalization of isotropic swelling due to arbitrary stimulus and proposed the Normalized Extended Temperature Expansion Model for its representation. ${ }^{1,21-23}$ Various responsivenesses are described therein, including thermal, electromagnetic, (bio-)chemical and mechanical responses. The output signal of the hydrogel is the local swelling, denoted by the Hencky strain $\varepsilon_{H}$. Please note that not all possible stimuli lead to isotropic material response. However, the anisotropic swelling affects only the geometric/spatial realization of logic elements. The intrinsic logic itself is a geometry independent material behavior.

After the definition of continuous input and output values, a threshold definition is performed. After analyzing the swelling behavior of a hydrogel material, thresholds to identify the binary high (1) and low (0) value of the input and output signals must be defined. In the normalized representation, they can be chosen e.g. as $80 \%$ values, see figure 4 . However, a sigmoid shape in all sensitivities is needed to clearly identify the reference point (inflexion point) and to use fixed percentages. In the current work, arbitrary swelling thresholds are used to show how the logic can be derived. In analogy to microelectronics, a region of undefined output is found. This region is needed to clearly distinguish between high and low value when noise comes into play. 


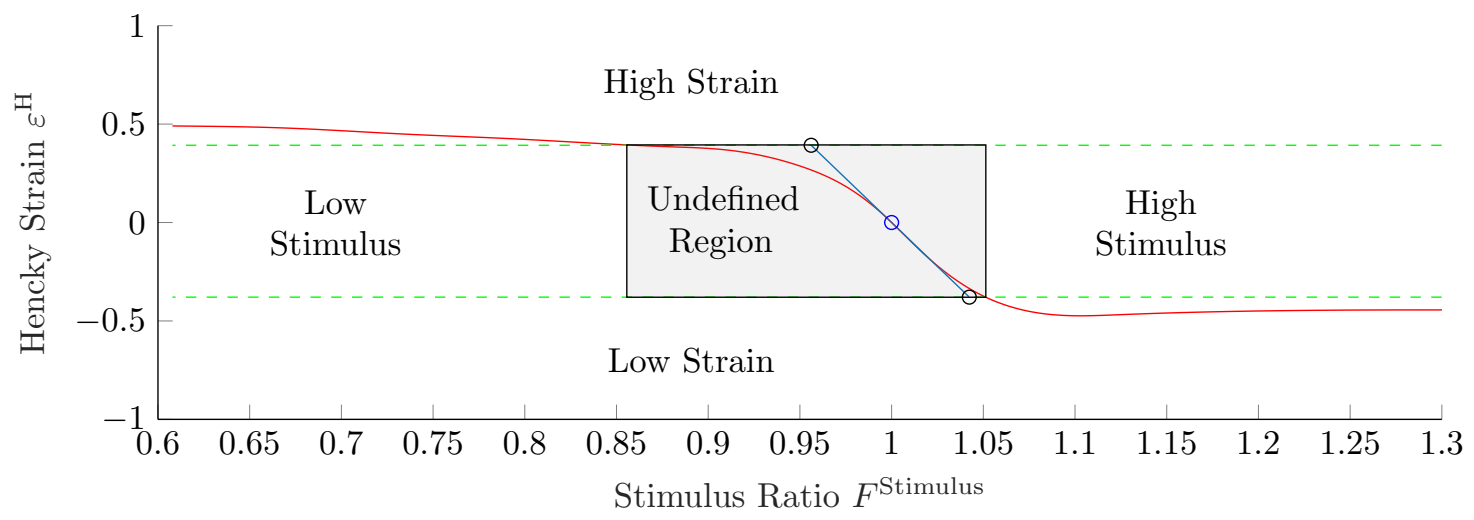

Figure 4: Definition of the thresholds, stimulus range and swelling range. The data is taken from our previous work. ${ }^{24}$ The simple setup forms a NOT gate.

The output in digitalized form can occur in three regions: For strain $\varepsilon^{\mathrm{H}} \leq \varepsilon^{\mathrm{H}}$, lower threshold it is low (0), for $\varepsilon^{\mathrm{H}} \geq \varepsilon^{\mathrm{H}}$, upper threshold it is high (1) and for every other value it is undefined (u).

From the intersection of the output thresholds and the borders of the undefined region, the stimulus thresholds are derived, see figure 4. The input is therefore digitalized so that it is low for $S \leq S^{\text {lower threshold, high for }}$ $S \geq S^{\text {upper threshold }}$ and undefined for every other value. Here, $S$ is used to denote the stimulus ratio $F^{\text {Stimulus }}$ for sake of simplicity. In the later section, the stimulus ratios for multiple input stimuli are named e.g. $S=p H$ for the stimulus ratio of the $\mathrm{pH}$-value.

Mechanical bistability mechanisms or hysteresis effects can be used to avoid the undefined region in the output of the mechanism in which the logic element is embedded, see section 3.

\subsection{Logic output behavior}

After the definition of thresholds, the binary response to any stimulus can be found. In the current work, we focus on an interpretation according to Boolean logic. However, other logic concepts like the three-valued Lukasiewicz-logic or unconventional computation concepts might also be appropriate.

For the analysis of the system's response to a number of stimuli $S_{i}$, the combinations can be arrayed using the disjunction $(\mathrm{OR} \vee$ ) of conjunction-pairs $(\mathrm{AND} \wedge$ ) in their original or negated $(\mathrm{NOT}-)$ form. The output $O$ is then represented e.g. by the disjunctive normal form

$$
O=\cup_{i} \overline{\left(\cap_{j}\left(S_{i}, S_{j}\right)\right)}
$$

When the logic table is at hand, the disjunctive normal form can directly be formed from rows that yield a 1 . Simplification of an unknown logical output behavior with DEMORGAN's rules provides an insight into the logic gate type of a material.

The actual placement of the hydrogel inside a mechanism can lead to a different output behavior of the overall setup. For example, when a hydrogel is placed inside a channel, ${ }^{25}$ a high swelling (1) leads to a blocking and therefore low flow (0) through the valve. This is a negating (NOT) behavior. However, from the viewpoint of material logic, the actual output is always the swelling. In technical applications such as the window-opener (see section 3), the amplification mechanism can also have its own negating behavior.

In the present work, we analyze two different materials from literature. The disjunctive normal forms for the overall logic gate behavior are derived according to equation (2) directly from the logic tables. 
Case I: Salt concentration and temperature As a first example, we take the swelling behavior of a semiinterpenetrating network hydrogel called [net-P(AMPS-co-NiPAAm)]-sipn-PAMPS. The composition, synthesis and characterization of this material are described in detail in the works of BINDER et al. ${ }^{26,27}$ and in our previous work, ${ }^{28}$ where we also give further insights into multi-sensitive behavior. The material is sensitive to sodium salt concentration and temperature.

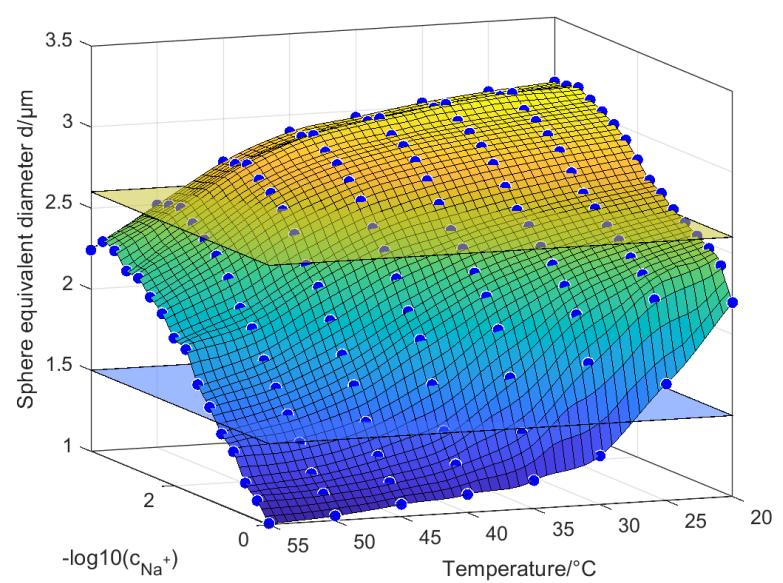

(a) Swelling surface for Case I (rotated for better visibility)

\begin{tabular}{c|c|c}
$-\log _{10}\left(c_{N a^{+}}\right)$ & $\vartheta$ & $O$ \\
\hline 0 & 0 & 0 \\
0 & 1 & 0 \\
1 & 0 & 0 \\
1 & 1 & 1
\end{tabular}

(b) Logic table for Case I

Figure 5: Hydrogel with AND behavior. Please note that the sodium ion stimulus is normalized in the same way as the $\mathrm{pH}$ value is defined for hydrogen ions. The sphere equivalent diameter $d$ represents the diameter of a sphere which swells and deswells; however, the swelling behavior can also be represented by the HENCKY-strain according to Figure 4.

The input signals are $S_{1}=-\log _{10}\left(c_{\mathrm{Na}^{+}}\right)$and $S_{2}=\vartheta$. In Figure 5 , the thresholds are denoted as planes with $d^{\text {threshold, upper }}=2.6 \mu \mathrm{m}$ and $d^{\text {threshold, lower }}=1.5 \mu \mathrm{m}$ The disjunctive normal form derived from the logic table is

$$
O=\left(-\log _{10}\left(c_{N a^{+}}\right) \wedge \vartheta\right)
$$

The type of the logic gate is therefore a simple AND gate. Please note that by changing the upper threshold to $2 \mu \mathrm{m}$, an OR gate behavior can be found. This can easily be achieved by changing the part's integration or changing the amplification mechanism.

Case II: Sugar concentration and pH-value A sample swelling behavior of bisensitive hydrogel copolymers is presented by Kim et al. ${ }^{29}$ The samples are made by copolymerization of acrylamide (AAm) with methacrylamidophenylboronic acid (MPBA). The swelling response to sugar molecules is due to binding reactions with the PBA. These can be glucose and other polyols that contain cis-diols, like fructose. The synthesized poly(MPBAco-AAm) hydrogel samples were immersed in aqueous buffer solutions of different $\mathrm{pH}$ value from $\mathrm{pH} 4$ to $\mathrm{pH}$ 10 at room temperature. In order to obtain the second sensitivity, fructose (Case IIa) or glucose (Case IIb) concentrations between 0 and $20 \mathrm{mM}$ were added. ${ }^{29}$ The equilibrium swelling curves for the bisensitive hydrogel systems fructose-pH and glucose-pH can also be used for a monosensitive sensor or actuator setup by keeping the other input constant.

Depending on the type of sugar - fructose or glucose - the swelling behavior and consequent logic output differs. It can be seen as $\mathrm{pH}$ - and fructose-sensitive (Case IIa), see figure 6. Input signals are $S_{1}=p H$ and $S_{2}=c_{\mathrm{F}}$.

The disjunctive normal form derived from the logic table is

$$
O=\left(\overline{c_{\mathrm{F}}} \wedge p H\right) \vee\left(c_{\mathrm{F}} \wedge p H\right)
$$




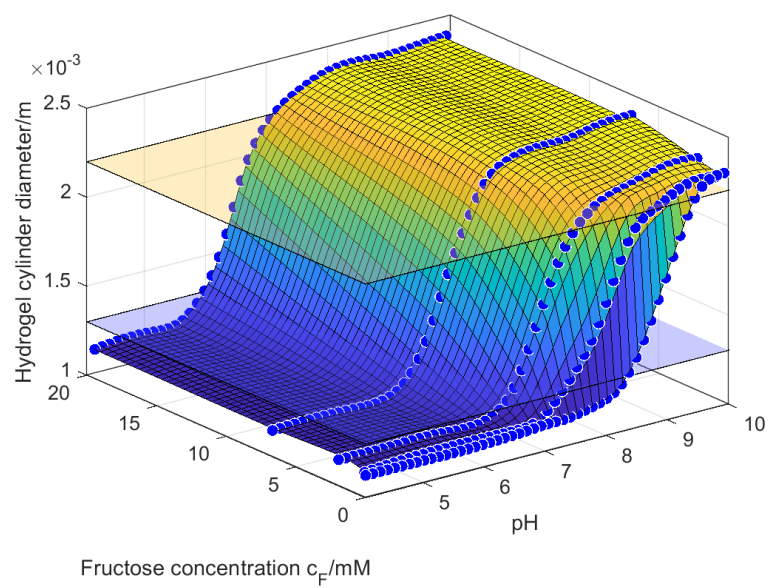

(a) Swelling surface for Case IIa

\begin{tabular}{c|c|c}
$c_{\mathrm{F}}$ & $p H$ & $O$ \\
\hline 0 & 0 & 0 \\
0 & 1 & 1 \\
1 & 0 & 0 \\
1 & 1 & 1
\end{tabular}

(b) Logic table for Case IIa

Figure 6: Case IIa: Hydrogel acting as an equal pH element under fructose stimulus.

and can be simplified to $O=p H$. The output signal is therefore independent from one of the input signals (fructose concentration) and an equal $\mathrm{pH}$ (identity) element is identified.

The same hydrogel can be used with other sugar molecules (Case IIb). It is then bisensitive to $\mathrm{pH}\left(S_{1}=p H\right)$ and glucose $\left(S_{2}=c_{\mathrm{G}}\right)$ concentration as presented in figure 7 .

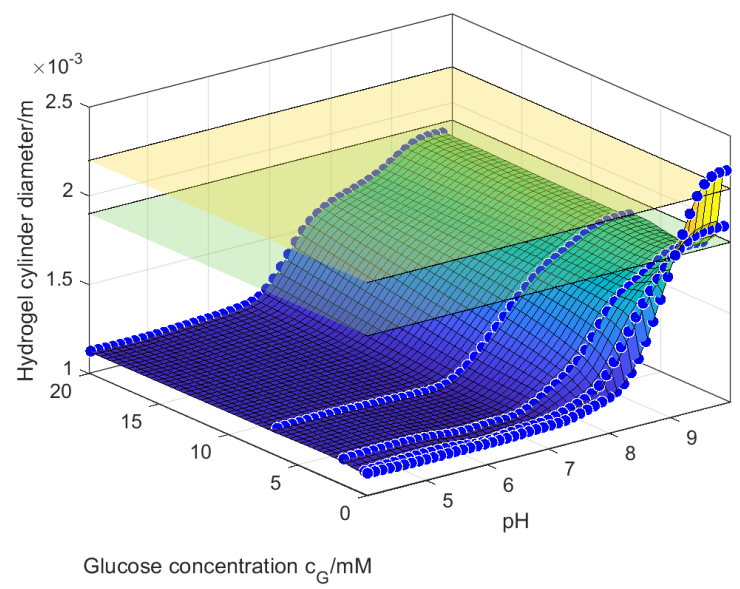

(a) Swelling surface for Case IIb

\begin{tabular}{c|c|c}
$c_{\mathrm{G}}$ & $p H$ & $O$ \\
\hline 0 & 0 & 0 \\
0 & 1 & 1 \\
1 & 0 & 0 \\
1 & 1 & 0
\end{tabular}

(b) Logic table for Case IIb

Figure 7: Case IIb: Hydrogel with inhibit behavior under glucose stimulus.

With the chosen thresholds, another logic form can be found, which is

$$
O=\left(\overline{c_{\mathrm{G}}} \wedge p H\right) .
$$

Please note that in this case, the region of high value is only in a very small glucose concentration range, the actual switching behavior is therefore in a limited stimulus range. The overall switching behavior translates to an inhibition gate: A low $\mathrm{pH}$ value inhibits the concentration switching behavior of this hydrogel. 


\begin{tabular}{l|l|l}
$I$ & $\vartheta$ & $O$ \\
\hline 0 & 0 & 0 \\
0 & 1 & 1 \\
1 & 0 & 0 \\
1 & 1 & 0
\end{tabular}

Table 1: Logic table of the task described in Figure 1. The logic combination of low light level (light intensity $I \downarrow$ below the lower threshold) and high temperature level (temperature $\vartheta \uparrow$ above the upper threshold) translates into an inhibit gate behavior.

To obtain materials that are better suited for the use in the framework of material logic, the hydrogels must be especially designed for this purpose. The possibilities of combining individual swelling behaviors of polymers by (i) co-polymerization or (ii) synthesis of IPNs can be used for this. ${ }^{30}$ The application of layer-systems with different monosensitive hydrogels offers another approach to design intelligent (meta-)materials for this purpose.

Please note that to date, there is no adequate material for the proposed automatic window-opener to be found in literature. The cooperation with chemists to synthesize and characterize the adequate material, as well as the experimental realization will be part of our future works.

\section{DESIGN OF THE AUTOMATIC WINDOW-OPENER}

An active material that offers the adequate responsiveness to light stimulus and temperature stimulus is included into a mechanism that handles a window pane. In its most simple form, the mechanism can be realized as presented in Figure 8. Other realizations can be e.g. cantilevers ${ }^{31}$ or minimum energy structures.

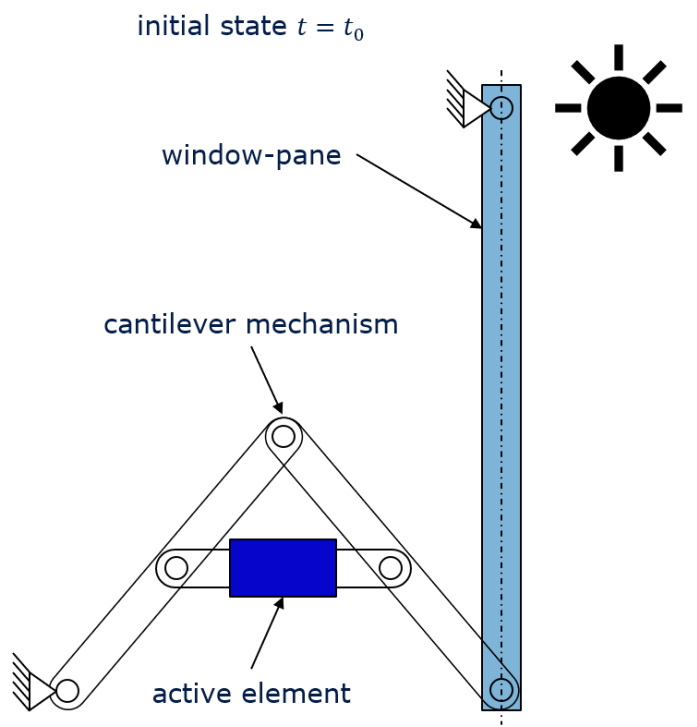

(a) Mechanism in initial state for high light level and arbitrary temperature level.

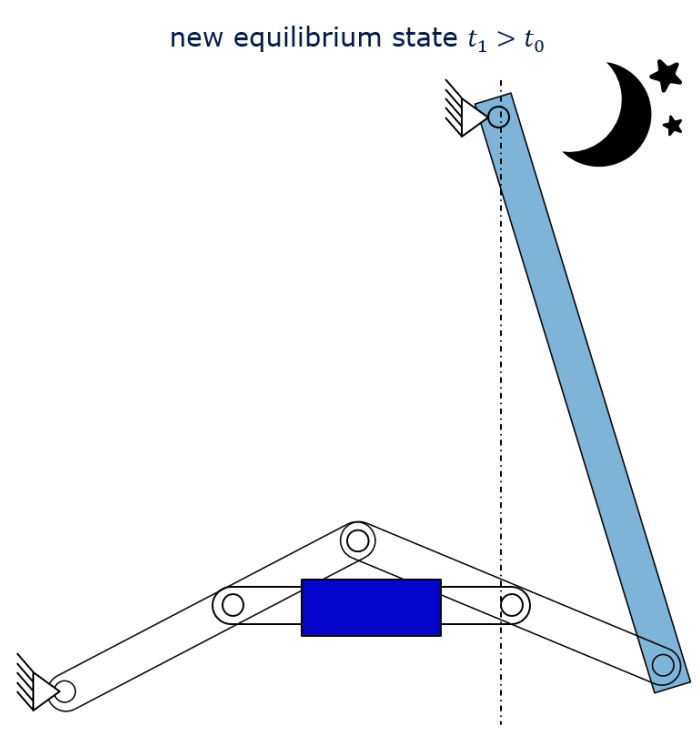

(b) Mechanism in new equilibrium state for low light level and high temperature level.

Figure 8: Simple mechanism that reacts according to the combination of stimuli.

The inner workings of the active element can be realized in various ways, such as applying buckling mechanisms $^{32,33}$ or mechanical 3D meta-materials. ${ }^{34}$ The main criterion is bistability in longitudinal direction that overcomes the undefined swelling zone described in Figure 4. The logic behavior according to the task from Figure 1 is an inhibit gate, see Table 1. This is the same logic behavior as shown in Case IIb, see 7 . 
The active element of the actuator can also be thought as a topology of inhomogeneous hydrogels filaments. ${ }^{35}$ These filaments can be manufactured either using a degradable scaffold through additive manufacturing techniques and 3D printing or using a combination of filament extrusion and textile braiding methods. ${ }^{36-38}$ The inhomogeneity of the hydrogels predefine the direction of the deformation of the filaments during the swelling process. In this context, two types of hydrogel with different swelling properties can be combined in order to provide the necessary force-displacement characteristics of the actuator. ${ }^{39}$ Specifically, due to the fact that current hydrogels provide high displacement due to the high swelling properties, and low forces, it is important to focus on unidirectional deformation by using lever-type techniques. ${ }^{40}$ In a further step of the material development, new types of hydrogels with a higher force-displacement ratio would be beneficial in these kind of applications.

\section{CONCLUSION AND OUTLOOK}

In the current work, we have presented the definition of the environment of an active material and how environments influence those materials. We have shown the concept of material logic behavior of multisensitive active materials for the example of hydrogels in an automatic window-opener. In our future works, we will present a specific material and specific setup for this application according to the presented concept.

\section{ACKNOWLEDGMENTS}

According to the CRediT system, the authors contributed to this publication as follows: AE: conceptualization, data curation, formal analysis, investigation, methodology, validation, visualization, writing - original draft, writing - review \& editing; ME: conceptualization, methodology, writing - review \& editing; AF: methodology, writing - review \& editing; MG: writing - review \& editing, resources, supervision, funding acquisition, project administration; TW: writing - review \& editing, resources, supervision, funding acquisition, project administration. We thank for the financial funding of the Dresden Center for Intelligent Materials (DCIM) by the Free State of Saxony and TU Dresden.

\section{REFERENCES}

[1] Ehrenhofer, A., Elstner, M., and Wallmersperger, T., "Normalization of hydrogel swelling behavior for sensoric and actuatoric applications," Sensors and Actuators B: Chemical 255(Part 2), 1343 - 1353 (2018).

[2] Gerlach, G. and Arndt, K.-F., [Hydrogel sensors and actuators: Engineering and technology], vol. 6, Springer Science \& Business Media (2009).

[3] Modler, N., Winkler, A., Filippatos, A., Weck, D., and Dannemann, M., "Function-integrative lightweight engineering-design methods and applications," Chemie Ingenieur Technik 92(7), 949-959 (2020).

[4] Pavlogeorgatos, G., "Environmental parameters in museums," Building and Environment 38(12), 1457-1462 (2003).

[5] Tang, J. W., "The effect of environmental parameters on the survival of airborne infectious agents," Journal of the Royal Society Interface 6(suppl_6), S737-S746 (2009).

[6] Srbinovska, M., Gavrovski, C., Dimcev, V., Krkoleva, A., and Borozan, V., "Environmental parameters monitoring in precision agriculture using wireless sensor networks," Journal of cleaner production 88, 297307 (2015).

[7] Mota Soares, C. A., Mota Soares, C. M., and Freitas, M. J., [Mechanics of composite materials and structures], vol. 361, Springer Science \& Business Media (2013).

[8] Newnham, R. E., [Ferroelectric Sensors and Actuators: Smart Ceramics], 363-380, Birkhäuser Basel, Basel (1993).

[9] Carpi, F., [Electromechanically Active Polymers: A Concise Reference], Springer (2016).

[10] Bar-Cohen, Y., [Electroactive Polymers as Artificial Muscles: Capabilities, Potentials and Challenges], ch. 23, 188-196, American Society of Civil Engineers (2000).

[11] Otero, T. F., Sanchez, J. J., and Martinez, J. G., "Biomimetic dual sensing-actuators based on conducting polymers. galvanostatic theoretical model for actuators sensing temperature," The Journal of Physical Chemistry B 116(17), 5279-5290 (2012).

[12] Leo, D. J., [Engineering analysis of smart material systems], John Wiley \& Sons (2007). 
[13] Mazaheri, H., Baghani, M., and Naghdabadi, R., "Inhomogeneous and homogeneous swelling behavior of temperature-sensitive poly-(n-isopropylacrylamide) hydrogels," Journal of Intelligent Material Systems and Structures 27(3), 324-336 (2016).

[14] Otero, T., Martinez, J., and Arias-Pardilla, J., "Biomimetic electrochemistry from conducting polymers. a review: artificial muscles, smart membranes, smart drug delivery and computer/neuron interfaces," Electrochimica Acta 84, 112-128 (2012).

[15] Ehrenhofer, A., Wallmersperger, T., and Richter, A., "Simulation of controllable permeation in pnipaam coated membranes," in [Proc. SPIE 9800, Behavior and Mechanics of Multifunctional Materials and Composites 2016], Proc. SPIE 980016 (2016).

[16] Chatterjee, S. and Hui, P. C.-l., "Stimuli-responsive hydrogels: An interdisciplinary overview," Hydrogels-Smart Materials for Biomedical Applications (2018).

[17] Smith, S. W. et al., "The scientist and engineer's guide to digital signal processing," (1997).

[18] Crama, Y. and Hammer, P. L., [Boolean functions: Theory, algorithms, and applications], Cambridge University Press (2011).

[19] Henke, E.-F. M., Schlatter, S., and Anderson, I. A., "A soft electronics-free robot," arXiv preprint arXiv:1603.05599 (2016).

[20] Wilson, K. E., Henke, E.-F. M., Slipher, G. A., and Anderson, I. A., "Rubbery logic gates," Extreme Mechanics Letters 9, 188-194 (2016).

[21] Ehrenhofer, A. and Wallmersperger, T., "A normalization concept for smart material actuation by the example of hydrogels," PAMM 18(1), e201800176 (2018).

[22] Ehrenhofer, A., Mieting, A., Pfeil, S., Mersch, J., Cherif, C., Gerlach, G., and Wallmersperger, T., "An automatically rainproofing bike helmet through light-sensitive hydrogel meshes: design, modeling and experiments," in [Electroactive Polymer Actuators and Devices (EAPAD) XXII], Bar-Cohen, Y., ed., 11375, 47 - 58, International Society for Optics and Photonics, SPIE (2020).

[23] Ehrenhofer, A. and Wallmersperger, T., "Shell-forming stimulus-active hydrogel composite membranes: Concept and modeling," Micromachines 11(6), 541 (2020).

[24] Ehrenhofer, A., Bingel, G., Paschew, G., Tietze, M., Schröder, R., Richter, A., and Wallmersperger, T., "Permeation control in hydrogel-layered patterned pet membranes with defined switchable pore geometry experiments and numerical simulation," Sensors and Actuators B: Chemical 232, 499-505 (2016).

[25] Paschew, G., Schreiter, J., Voigt, A., Pini, C., Chávez, J. P., Allerdißen, M., Marschner, U., Siegmund, S., Schüffny, R., Jülicher, F., et al., "Autonomous chemical oscillator circuit based on bidirectional chemicalmicrofluidic coupling," Advanced Materials Technologies 1, 1 (2016). Project: Membranes.

[26] Binder, S., Krause, A. T., Voit, B., and Gerlach, G., "Bisensitive hydrogel with volume compensation properties for force compensation sensors," IEEE Sensors Letters 1(6), 1-4 (2017).

[27] Binder, S., Zschoche, S., Voit, B., and Gerlach, G., "Semi-interpenetrating polymer networks based on nisopropylacrylamide and 2-acrylamido-2-methylpropane sulfonic acid for intramolecular force-compensated sensors," Journal of the Electrochemical Society 167(16), 167521 (2021).

[28] Ehrenhofer, A., Binder, S., Gerlach, G., and Wallmersperger, T., "Multisensitive swelling of hydrogels for sensor and actuator design," Advanced Engineering Materials (2020).

[29] Kim, A., Mujumdar, S. K., and Siegel, R. A., "Swelling properties of hydrogels containing phenylboronic acids," Chemosensors 2(1), 1-12 (2013).

[30] Krause, A., Zschoche, S., Rohn, M., Hempel, C., Richter, A., Appelhans, D., and Voit, B., "Swelling behavior of bisensitive interpenetrating polymer networks for microfluidic applications," Soft matter 12 (25), 5529-5536 (2016).

[31] Ehrenhofer, A. and Wallmersperger, T., "Hydrogels for engineering: normalization of swelling due to arbitrary stimulus," in [Proc. SPIE 10163, Electroactive Polymer Actuators and Devices XIX], Proc. SPIE 10163, 1016321-15 (2017).

[32] Qiu, J., Lang, J. H., and Slocum, A. H., "A curved-beam bistable mechanism," Journal of microelectromechanical systems 13(2), 137-146 (2004).

[33] Hu, N. and Burgueño, R., "Buckling-induced smart applications: recent advances and trends," Smart Materials and Structures 24(6), 063001 (2015). 
[34] Kadic, M., Milton, G. W., van Hecke, M., and Wegener, M., "3d metamaterials," Nature Reviews Physics 1(3), 198-210 (2019).

[35] Ionov, L., "Hydrogel-based actuators: possibilities and limitations," Materials Today 17(10), 494-503 (2014).

[36] Hinton, T. J., Jallerat, Q., Palchesko, R. N., Park, J. H., Grodzicki, M. S., Shue, H.-J., Ramadan, M. H., Hudson, A. R., and Feinberg, A. W., "Three-dimensional printing of complex biological structures by freeform reversible embedding of suspended hydrogels," Science advances 1(9), e1500758 (2015).

[37] Jiang, Z., Diggle, B., Tan, M. L., Viktorova, J., Bennett, C. W., and Connal, L. A., "Extrusion 3d printing of polymeric materials with advanced properties," Advanced Science 7(17), 2001379 (2020).

[38] Askari, M., Naniz, M. A., Kouhi, M., Saberi, A., Zolfagharian, A., and Bodaghi, M., "Recent progress in extrusion 3d bioprinting of hydrogel biomaterials for tissue regeneration: A comprehensive review with focus on advanced fabrication techniques," Biomaterials science (2021).

[39] Jiang, H., Fan, L., Yan, S., Li, F., Li, H., and Tang, J., "Tough and electro-responsive hydrogel actuators with bidirectional bending behavior," Nanoscale 11(5), 2231-2237 (2019).

[40] Charpentier, V., Hannequart, P., Adriaenssens, S., Baverel, O., Viglino, E., and Eisenman, S., "Kinematic amplification strategies in plants and engineering," Smart Materials and Structures 26(6), 063002 (2017). 